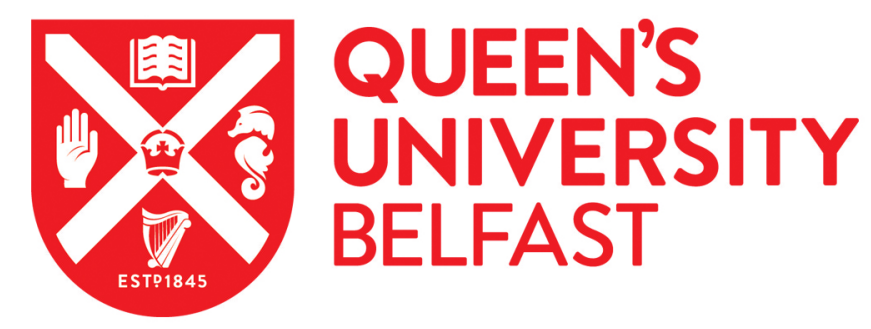

\title{
Approach to chaos in ultracold atomic and molecular physics: Statistics of near-threshold bound states for $\mathrm{Li}+\mathrm{CaH}$ and $\mathrm{Li}+\mathrm{CaF}$
}

Frye, M. D., Morita, M., Vaillant, C. L., Green, D. G., \& Hutson, J. M. (2016). Approach to chaos in ultracold atomic and molecular physics: Statistics of near-threshold bound states for $\mathrm{Li}+\mathrm{CaH}$ and $\mathrm{Li}+\mathrm{CaF}$. Physical Review A (Atomic, Molecular, and Optical Physics), 93(5), [052713].

https://doi.org/10.1103/PhysRevA.93.052713

Published in:

Physical Review A (Atomic, Molecular, and Optical Physics)

Document Version:

Peer reviewed version

Queen's University Belfast - Research Portal:

Link to publication record in Queen's University Belfast Research Portal

Publisher rights

@2016 American Physical Society

\section{General rights}

Copyright for the publications made accessible via the Queen's University Belfast Research Portal is retained by the author(s) and / or other copyright owners and it is a condition of accessing these publications that users recognise and abide by the legal requirements associated with these rights.

Take down policy

The Research Portal is Queen's institutional repository that provides access to Queen's research output. Every effort has been made to ensure that content in the Research Portal does not infringe any person's rights, or applicable UK laws. If you discover content in the Research Portal that you believe breaches copyright or violates any law, please contact openaccess@qub.ac.uk. 


\title{
The approach to chaos in ultracold atomic and molecular physics: statistics of near-threshold bound states for $\mathrm{Li}+\mathrm{CaH}$ and $\mathrm{Li}+\mathrm{CaF}$
}

\author{
Matthew D. Frye, Masato Morita, Christophe L. Vaillant, ${ }^{*}$ Dermot G. Green, ${ }^{\dagger}$ and Jeremy M. Hutson ${ }^{\ddagger}$ \\ Joint Quantum Centre (JQC) Durham-Newcastle, Department of Chemistry, \\ Durham University, South Road, Durham DH1 3LE, United Kingdom.
}

(Dated: April 22, 2016)

\begin{abstract}
We calculate near-threshold bound states and Feshbach resonance positions for atom + rigid-rotor models of the highly anisotropic systems $\mathrm{Li}+\mathrm{CaH}$ and $\mathrm{Li}+\mathrm{CaF}$. We perform statistical analysis on the resonance positions to compare with the predictions of random matrix theory. For $\mathrm{Li}+\mathrm{CaH}$ with total angular momentum $J=0$ we find fully chaotic behavior in both the nearest-neighbor spacing distribution and the level number variance. However, for $J>0$ we find different behavior due to the presence of a nearly conserved quantum number. $\mathrm{Li}+\mathrm{CaF}(J=0)$ also shows apparently reduced levels of chaotic behavior despite its stronger effective coupling. We suggest this may indicate the development of another good quantum number relating to a bending motion of the complex. However, continuously varying the rotational constant over a wide range shows unexpected structure in the degree of chaotic behavior, including a dramatic reduction around the rotational constant of $\mathrm{CaF}$. This demonstrates the complexity of the relationship between coupling and chaotic behavior.
\end{abstract}

\section{INTRODUCTION}

There is currently great interest in the behavior of atoms and molecules at ultracold temperatures. Work in this field focussed initially on simple systems, which are amenable to detailed control by tuning with applied fields [1]. Particular success has been achieved with ultracold collisions and high-lying bound states of pairs of alkalimetal atoms, where a combination of experiment and theory has produced a detailed understanding for a variety of different systems [2-4]. This understanding has been used to study solitons in Bose-Einstein condensates [5], diatomic molecule formation [6], Efimov states $[7,8]$ and many other phenomena. However, attention is now turning to increasingly complex systems, such as collisions of high-spin atoms [9-14] and ground-state molecules [1519]. The theory needed for a full description of these systems is often prohibitively difficult; for example, two erbium atoms interact on 91 potential curves [20], so that it is unrealistic to fit precise potentials to experimental results in the manner that has been so successful for alkali dimers [2-4]. In these circumstances, it is possible that the dynamics are stochastic, with levels described by random matrix theory $[21,22]$. These ideas were first developed in the theory of compound nuclei [23, 24], and underlie current thinking about the nature of quantum chaos.

A qualitative understanding of the dynamics of these highly complex systems is important for both theorists and experimentalists. For a simple system, theory can

\footnotetext{
* c.l.j.j.vaillant@durham.ac.uk

$\dagger$ Present address: Centre for Theoretical Atomic, Molecular and Optical Physics, School of Mathematics and Physics, Queen's University Belfast, Belfast BT7 1NN, Northern Ireland, United Kingdom; d.green@qub.ac.uk

¥ Author to whom correspondence should be addressed; j.m.hutson@durham.ac.uk
}

in principle give a complete description, but for a fully chaotic system specific predictions are likely to be impossible. For example, it is unlikely to be possible to map out the near-threshold bound states navigated by Feshbach molecules except empirically [14]. If a collision is chaotic in nature, the collision complex will ergodically explore the entire phase-space and the two collision partners may be trapped together for a long time. In ultracold collisions, this may be long enough for a third body to collide with the complex, which is likely to lead to the loss of all the particles involved from the trap [25].

Random matrix theory (RMT) was first applied to ultracold collisions by Mayle et al., initially for atom+diatom collisions [25] and later for diatom+diatom collisions [26]. The first experimental demonstration of these ideas was provided by atom+atom collisions for Er+Er [20] and later Dy+Dy [27]. Several theoretical models have also been analyzed in terms of RMT $[20,27,28]$. Within RMT the archetypal model of quantum chaos is a matrix ensemble known as the Gaussian Orthogonal Ensemble (GOE) [21, 24] and the usual way to assess whether or not chaos is present in a level sequence is to compare various statistics to the expectations from the GOE. In particular, the BohigasGiannoni-Schmit conjecture [29] states that quantum systems whose classical analogues are chaotic will show the same level fluctuations as the GOE.

There are many statistics available to analyze level sequences (see for example chapter 16 of Mehta's book [21]) but work on ultracold collisions so far has focused on two of the simplest quantities: the distribution of nearestneighbor spacings (NNS) and the level number variance. In ultracold collisions, the levels (or resonances) concerned are typically observed as a function of magnetic field rather than energy. Erbium and dysprosium have been found to have NNS distributions and number variances that are intermediate between those expected for chaotic and non-chaotic systems [20, 27]. A re-analysis of the erbium results [30] showed that the deviations 
from chaotic predictions might arise because some narrow resonances are not observed in the experiment. A comparable degree of chaos has recently been found in coupled-channel calculations on the remarkably simple system $\mathrm{Yb}\left({ }^{1} \mathrm{~S}\right)+\mathrm{Yb}\left({ }^{3} \mathrm{P}\right)[31]$. However, similar calculations on $\mathrm{Li}+\mathrm{Er}$ found statistics consistent with a random but non-chaotic level distribution [32].

Atom+diatom systems provide excellent prototype systems to investigate chaotic behavior. If vibrational excitation and electron spins are neglected, the systems have two internal degrees of freedom; this is the minimum for classical chaos and probably also for a quantum system to follow the predictions of RMT [29]. There are well-developed formalisms for treating collisions [33] and near-threshold bound states $[34,35]$ in atom+diatom systems, and readily available programs that efficiently perform the necessary calculations $[36,37]$. There are many atom+diatom systems that are of interest at low temperatures, including $\mathrm{Rb}+\mathrm{KRb}[16], \mathrm{N}+\mathrm{NH}[38]$ and $\mathrm{Li}+\mathrm{CaH}[39]$.

The purpose of the present paper is to investigate quantum chaos in atom+diatom collisions. We investigate the statistics of levels at and near threshold for atom + rigid-rotor models of the highly anisotropic systems $\mathrm{Li}+\mathrm{CaH}$ and $\mathrm{Li}+\mathrm{CaF}$, and compare them with the predictions of RMT. For simplicity, we neglect electron and nuclear spins. For $\mathrm{Li}+\mathrm{CaH}$ with zero total angular momentum we find excellent agreement with RMT. However for higher angular momentum we find quite different behavior which we attribute to the presence of a nearly conserved quantum number. $\mathrm{Li}+\mathrm{CaF}$ has a larger ratio of anisotropy to rotational constant than $\mathrm{Li}+\mathrm{CaH}$, and in this sense it is more strongly coupled, but we actually find it is less strongly chaotic, even for zero angular momentum. This shows that increasing coupling does not always make a system more chaotic and may, in some circumstances, return order to the system. We analyze statistics as a function of a continuously varying rotational constant, interpolating between $\mathrm{CaH}$ and $\mathrm{CaF}$ and beyond, and find complex behavior with apparently fluctuating levels of chaoticity.

\section{POTENTIAL ENERGY SURFACE}

The interaction between $\mathrm{Li}\left({ }^{2} \mathrm{~S}\right)$ and $\mathrm{CaH} / \mathrm{CaF}\left({ }^{2} \Sigma\right)$ gives rise to singlet and triplet electronic states of ${ }^{1} \mathrm{~A}^{\prime}$ and ${ }^{3} \mathrm{~A}^{\prime}$ symmetries. For sympathetic cooling in an external magnetic field, $\mathrm{Li}$ and $\mathrm{CaH} / \mathrm{CaF}$ would be prepared in magnetically trappable spin-stretched states, in which all the quantum numbers for the projections of angular momentum onto the magnetic field direction have their maximum values. Such collisions occur primarily on the potential energy surface for the ${ }^{3} \mathrm{~A}^{\prime}$ state, so we use this surface in the present paper.

For $\mathrm{Li}+\mathrm{CaH}$, we use the ab initio ${ }^{3} \mathrm{~A}^{\prime}$ interaction potential calculated by Tscherbul et al. [40]. For $\mathrm{Li}+\mathrm{CaF}$, we carried out supermolecular coupled-cluster calcula- tions of the ${ }^{3} \mathrm{~A}^{\prime}$ surface using a spin-restricted open-shell version of the coupled-cluster method with single, double and non-iterative triple excitations $[\operatorname{RCCSD}(\mathrm{T})]$, which is implemented in the MOLPRO package [41]. We used the aug-cc-pVTZ basis sets for each atom [42-44]. Midbond functions (spdfg) were added at the midpoint between $\mathrm{Li}$ and $\mathrm{CaF}$. The counterpoise correction of Boys and Bernardi [45] was used to compensate for basis set superposition error. In all our calculations the $\mathrm{CaF}$ bond length was fixed at the experimentally determined equilibrium value for the free diatom, $1.9516 \AA$ [46].

The resulting ${ }^{3} \mathrm{~A}^{\prime}$ potential energy surfaces are shown in Fig. 1, and it may be seen that they are strongly anisotropic, with a deep well $\left(7063 \mathrm{~cm}^{-1}\right.$ for $\mathrm{CaH}$ and $7258 \mathrm{~cm}^{-1}$ for $\mathrm{CaF}$ ) at slightly bent $\mathrm{Li}-X$-Ca geometries. The overall behavior of the two surfaces is similar, though the $\mathrm{Li}+\mathrm{CaF}$ interaction is slightly stronger and more anisotropic.

\section{BOUND-STATE CALCULATIONS AND STATISTICS}

When considering whether a system is chaotic, statistical analysis would usually be performed on a series of levels in energy with the Hamiltonian defining the system fixed. However, the underlying Random Matrix Theory concerns distributions taken over an ensemble of different Hamiltonians. The assumption that the distributions over energy (for a single Hamiltonian) are the same as those over Hamiltonians is known as spectral ergodicity [47]; this is a property of RMT but not necessarily of real systems. In recent ultracold collision studies $[20,27,28]$, statistical analysis was performed on a series of zero-energy resonance positions as magnetic field was varied. Such experiments sample many different Hamiltonians - albeit in a much more limited and structured way than RMT - and so may provide a more authentic comparison with RMT than an energy spectrum would, even if it was available. In place of the spectral ergodicity hypothesis, we now need only to assume that different Hamiltonians are sampled in a representative way.

Performing calculations on $\mathrm{Li}+\mathrm{CaH}$ and $\mathrm{Li}+\mathrm{CaF}$ in magnetic fields is a difficult and computationally expensive task, which is beyond the scope of this paper but will be investigated in the future. Instead, we vary the potential by an overall scaling factor $\lambda$ [48-50], which also varies the Hamiltonian but without the theoretical complexities or computational expense associated with magnetic fields [51]. In this respect $\lambda$ may be considered as a substitute for magnetic field. If a system shows signs of chaos with respect to $\lambda$, it is reasonable to label it chaotic (under the approximations made for the dynamics).

The theory of atom-diatom interactions is well established $[33,34]$ and will not be repeated here. In the present work, we carry out close-coupling calculations in which the diatomic molecule is treated as a rigid rotor 

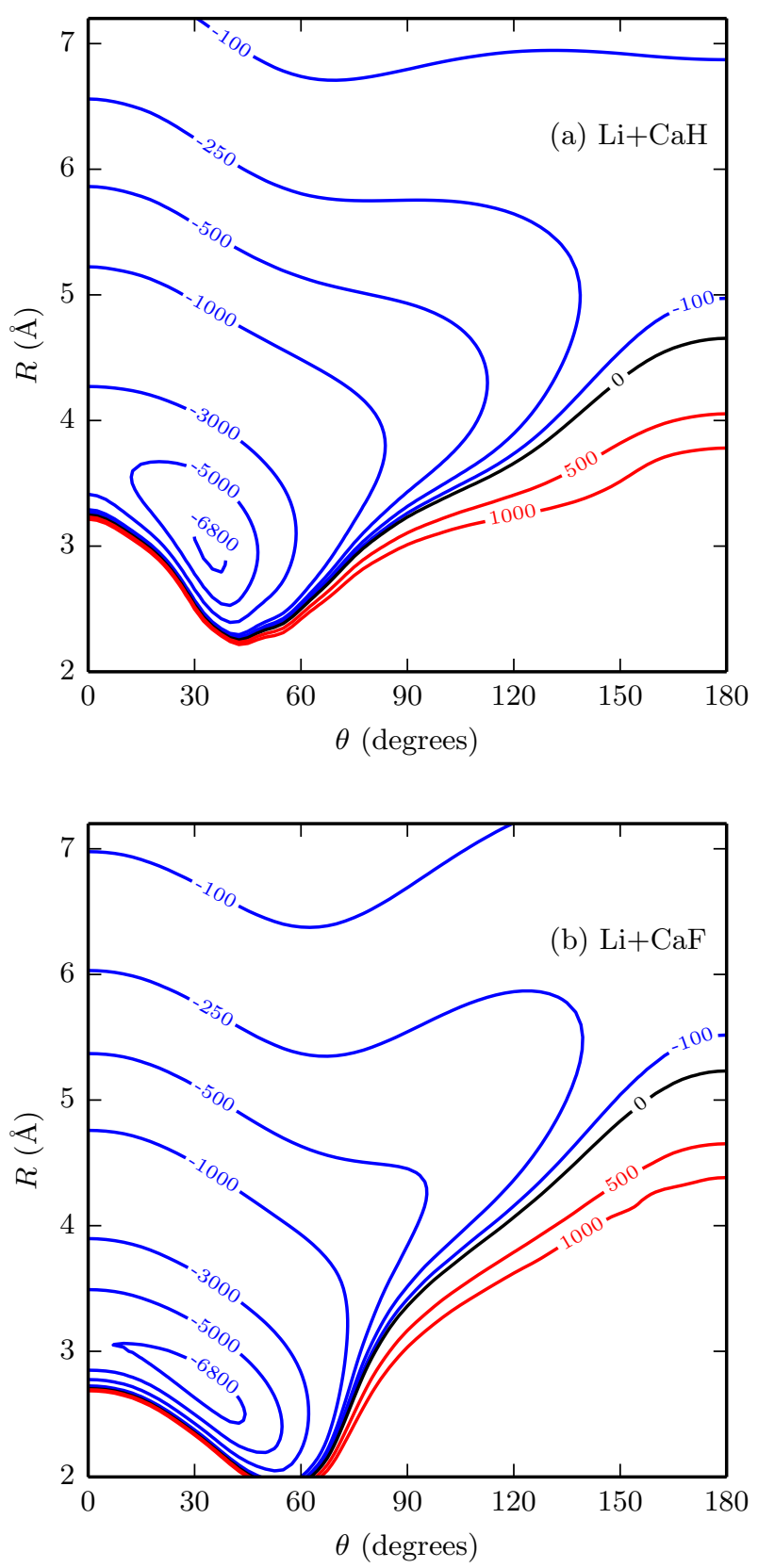

FIG. 1. (Color online) The ab initio ${ }^{3} \mathrm{~A}^{\prime}$ interaction potential for (a) $\mathrm{Li}+\mathrm{CaH}$ and (b) $\mathrm{Li}+\mathrm{CaF}$. Contours are labeled in $\mathrm{cm}^{-1} \cdot \theta=0^{\circ}$ corresponds to the Li-H(F)-Ca geometry.

and its rotation $j$ is coupled to the end-over-end rotation of the collision complex $L$ to give the total angular momentum $J=j+L$. We use coupled-channel propagation methods [35] to locate bound states at threshold. We have modified the program FIELD [52] to locate bound states as a function of $\lambda$ rather than electric or magnetic field. We use the hybrid log-derivative propagator of Alexander and Manolopoulos [53]. Since the diatom rotational constants, $b$, are very small $\left(b_{\mathrm{CaH}}=4.2766\right.$ $\mathrm{cm}^{-1}[40]$ and $\left.b_{\mathrm{CaF}}=0.339 \mathrm{~cm}^{-1}[54]\right)$, we need very large basis sets of diatom rotational functions for convergence. Unless otherwise stated, we use basis functions with rotational quantum numbers up to $j_{\max }=55$ and 120 for $\mathrm{CaH}$ and $\mathrm{CaF}$, respectively.

The real systems include diatom vibrations and electron and nuclear spins. The harmonic frequencies for $\mathrm{CaH}$ and $\mathrm{CaF}$ are 1298 and $589 \mathrm{~cm}^{-1}$, respectively. Since the well depth is significantly larger than this, there will be states from channels involving diatom vibrational excitation in the region around threshold, although they may be sparse in energy. These are neglected in our calculations. There will also be considerable extra density of levels due to the spin multiplicities, although it is not clear whether the spins will be fully involved in any chaotic dynamics or if they will be spectators. The present results are therefore for model systems, based on the real systems but not taking account of their full complexity.

The Gaussian Orthogonal Ensemble (GOE) is the standard RMT model for chaos in systems with time-reversalinvariant Hamiltonians. It is a set of $N \times N$ real symmetric matrices, with diagonal and off-diagonal elements described by probability distributions

$$
\begin{aligned}
P_{i i}\left(H_{i i}\right) & =C \exp \left(-H_{i i}^{2}\right), \\
P_{i j}\left(H_{i j}\right) & =C^{\prime} \exp \left(-2 H_{i j}^{2}\right),
\end{aligned}
$$

respectively, where $C$ and $C^{\prime}$ are normalization constants. The GOE has off-diagonal elements that are on the order of the spread of the diagonal elements and so for large $N$ are much larger than the average separation of diagonal elements. In the context of nearthreshold bound states or low-energy collisions, this can occur when the anisotropic terms in the interaction are comparable in magnitude to the depth of the isotropic potential, which determines the spread of diagonal elements. The highly anisotropic potentials of $\mathrm{Li}+\mathrm{CaH}$ and $\mathrm{Li}+\mathrm{CaF}$ would seem to fulfil this: the majority of the potential well is contained in the anisotropic terms of many thousands of $\mathrm{cm}^{-1}$, which are significantly larger than the depth of the isotropic potential; the latter is only 1260 $\mathrm{cm}^{-1}$ for $\mathrm{Li}+\mathrm{CaH}$. In contrast, the $\mathrm{Er}+\mathrm{Er}$ and $\mathrm{Dy}+\mathrm{Dy}$ potentials used in Ref. [27] have anisotropies around $10 \%$ of their well depths [55]. In this way it is perhaps not surprising that there are deviations from GOE predictions for $\mathrm{Er}+\mathrm{Er}$ and Dy+Dy, whereas we might at first sight expect better agreement for $\mathrm{Li}+\mathrm{CaH}$ and $\mathrm{Li}+\mathrm{CaF}$.

For the statistical analysis of the levels we follow the methods of Mehta [21] and Guhr et al. [22]. We denote our series of $n$ calculated level positions as $X_{i}$ for $i=1, \ldots, n[56]$. First we 'unfold' the level positions to remove any systematic variation in the density and to set the levels on a dimensionless scale with unit mean spacing. To do this we construct the cumulative spectral function, also known as the staircase function,

$$
S(X)=\sum_{i=1}^{n} \Theta\left(X-X_{i}\right)
$$


where $\Theta(x)$ is the Heaviside step function. We then fit a smooth function, $\xi(X)$, to $S(X)$ (in this paper, fitting with a quadratic is sufficient as the original staircase is already very nearly linear in $\lambda)$. The unfolded positions are then given as $\xi_{i}=\xi\left(X_{i}\right)$. The nearest-neighbor spacings (NNS) are given by $s_{i}=\xi_{i+1}-\xi_{i}$ for $i=1,2 . ., n-1$.

The NNS distribution is one of the main quantities of interest in statistical analysis. In particular, for the GOE the NNS distribution is very well approximated by the Wigner surmise [21],

$$
P_{\mathrm{WD}}(s)=\frac{\pi s}{2} \exp \left(-\frac{\pi s^{2}}{4}\right),
$$

more commonly known as the Wigner-Dyson distribution, whereas for a random (uncorrelated) level sequence the NNS distribution is expected to follow a Poisson distribution,

$$
P_{\mathrm{P}}(s)=\exp (-s) .
$$

Histograms of the NNS distribution provide a simple visual impression of the statistics. The Wigner-Dyson distribution exhibits strong level repulsion, dropping to zero at zero spacing, whereas the Poisson distribution peaks at zero spacing. The Wigner-Dyson distribution also falls to zero faster at large spacing than the Poisson distribution.

Real systems do not exactly follow either $P_{\mathrm{WD}}(s)$ or $P_{\mathrm{P}}(s)$. There are various formulas for interpolating between the two $[57,58]$. The most commonly used of these, despite its lack of rigorous foundation, is the Brody distribution [59],

$$
P_{\mathrm{B}}^{(\eta)}(s)=c_{\eta}(\eta+1) s^{\eta} \exp \left(-c_{\eta} s^{\eta+1}\right),
$$

where

$$
c_{\eta}=\left[\Gamma\left(\frac{\eta+2}{\eta+1}\right)\right]^{\eta+1},
$$

and $\eta$ is known as the Brody parameter. This is the NNS distribution that has been used in recent publications in ultracold physics and is the one that we use in this paper. We obtain a value of $\eta$ for a set of spacings by maximum likelihood estimation [60]. We maximise the likelihood function,

$$
L(\eta)=\prod_{i} P_{\mathrm{B}}^{(\eta)}\left(s_{i}\right),
$$

to find $\eta$. Its uncertainty is

$$
\sigma=\left(-\frac{d^{2}}{d \eta^{2}} \ln L(\eta)\right)^{-\frac{1}{2}}
$$

The fitted value for the Brody parameter quantifies the visual information seen in NNS histograms: Poisson statistics yield $\eta=0$ and chaotic statistics yield $\eta=1$. If the pattern of energy levels is in fact regular, the
NNS distribution is typically more strongly peaked than a Wigner-Dyson distribution [61], and so the fitted Brody parameter may be greater than unity. We do find some fitted values $\eta>1$, but they are consistent with $\eta=1$ within statistical uncertainties.

The NNS distribution by nature captures information only about short-range correlations but chaos is predicted to have effects over long ranges as well [21, 22]. Therefore we also consider the level number variance

$$
\Sigma^{2}(\Delta \xi)=\left\langle\hat{S}^{2}(\Delta \xi, \xi)\right\rangle-\langle\hat{S}(\Delta \xi, \xi)\rangle^{2},
$$

where $\hat{S}(\Delta \xi, \xi)$ counts the number of levels in the interval $[\xi, \xi+\Delta \xi]$ and the average is taken over the starting values $\xi$. This characterizes the spread in the numbers of levels in intervals of length $\Delta \xi$ and probes long-range correlations. It rises logarithmically for the GOE, rises linearly for Poisson statistics, and oscillates around a constant value for a regular system. While there have been some attempts to interpolate between Poissonian and GOE behaviors of the number variance, there is no direct analogue to the Brody distribution so we restrict ourselves to qualitative statements about the transition between the two limiting behaviors.

\section{RESULTS}

\section{A. $\mathbf{L i}+\mathbf{C a H}$}

We begin by analyzing $\mathrm{Li}+\mathrm{CaH}$ for total angular momentum $J=0$ and $0.7 \leq \lambda \leq 1.6$. Figure 2 shows the calculated level positions for $J=0$, the staircase function, a histogram of the NNS distribution, and the level number variance. This serves as an example of the statistical preparation and fitting described in section III; all further sequences were analyzed in the same way. The NNS distribution clearly shows the key features of a Wigner-Dyson distribution: linear repulsion at small spacing and a tail that dies off rapidly. The fitted Brody parameter, $\eta=1.08 \pm 0.10$, is consistent with GOE predictions, and the level number variance also follows the GOE prediction almost exactly. This Brody parameter may be compared with values in the region 0.5 to 0.7 found for Er+Er and Dy+Dy [27]. Our recent work on $\mathrm{Yb}+\mathrm{Yb}^{*}[31]$ also found Brody parameters around 0.7 at high magnetic field, except in cases with relatively low numbers of levels. The rigid-rotor model of $\mathrm{Li}+\mathrm{CaH}$ thus shows the clearest evidence yet found of chaotic behavior in ultracold collisions.

Figure 3 shows near-threshold bound states for $J=0$ for a small range of $\lambda$. The top panel shows bound states to a depth of $10 \mathrm{~cm}^{-1}$, where the levels interact and undergo avoided crossings with a wide variety of strengths. The lower panel is an expanded view showing a state with a long tail curving towards threshold. This state is crossed by several steeper states with avoided crossings of varying widths, including a crossing around $\lambda=1.007$ 

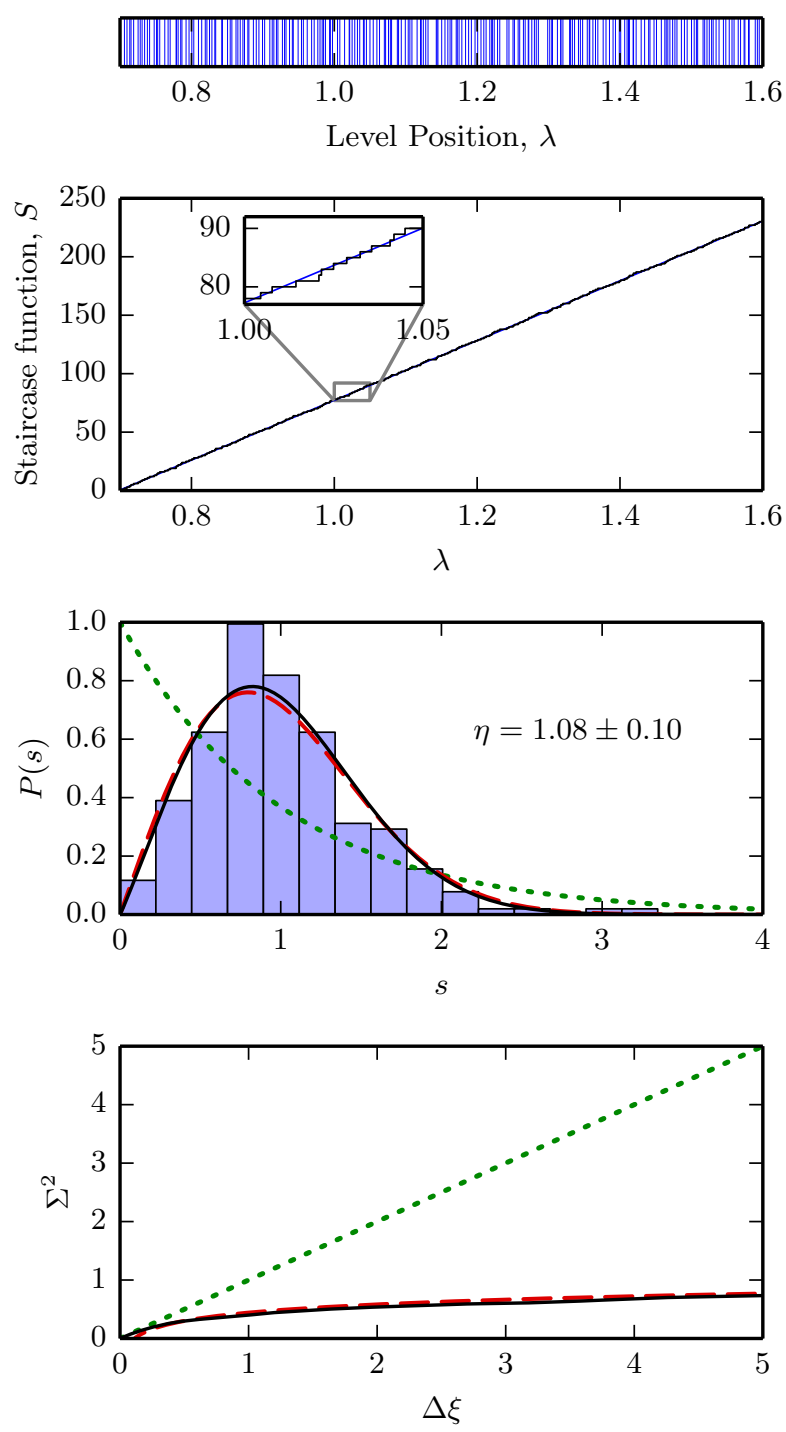

FIG. 2. (Color online) Statistical analysis of calculated levels of $\mathrm{Li}+\mathrm{CaH}(J=0,0.7 \leq \lambda \leq 1.6)$. From top: Level positions in $\lambda$; Staircase function, with fitted smooth function $\xi(X)$ in blue; Histogram of NNS distribution, green dotted and red dashed lines are Poisson and Wigner-Dyson distributions respectively, black line is Brody distribution using the fitted $\eta$; Level number variance, green dotted and red dashed lines are Poisson and GOE predictions respectively.

that appears quite broad on this scale. Bound states with these features have been observed experimentally in Dy+Dy [13], although in our case the state is much more deeply bound (by a factor of about 100 in the natural units defined by the asymptotic van der Waals interaction relevant to such states [1]). It is not a 'halo' state because its wave function is mostly inside the outer classical turning point [6], but its presence in $\mathrm{Li}+\mathrm{CaH}$ demonstrates that states with clear threshold behavior can persist across several avoided crossings even in a system with statistics close to the Wigner-Dyson limit.
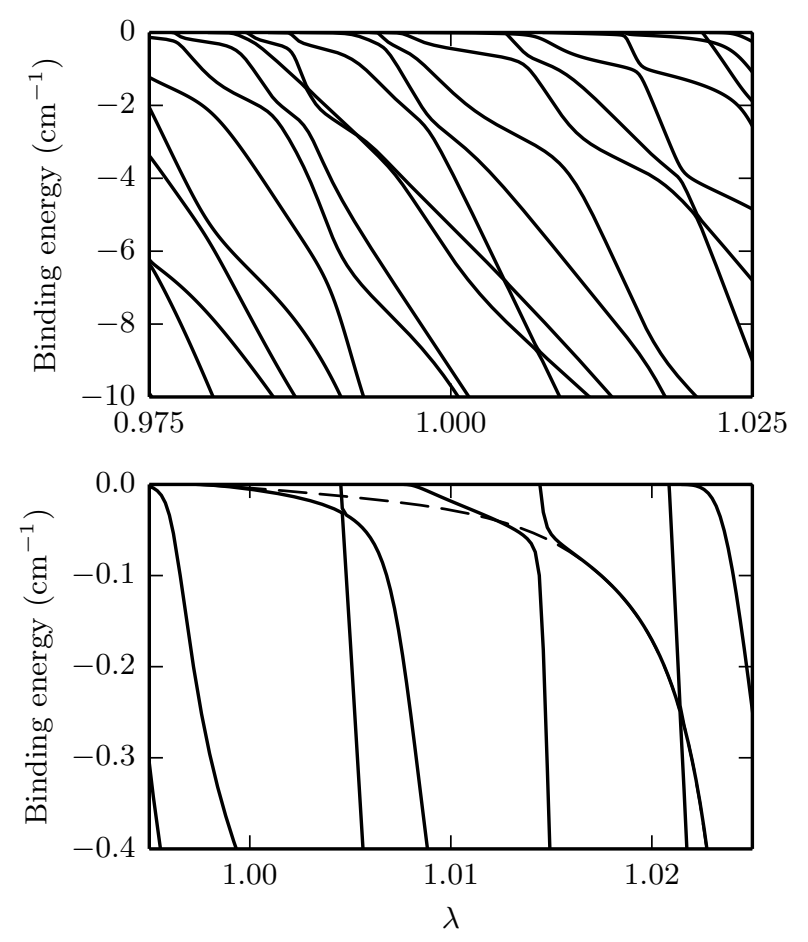

FIG. 3. Near-threshold bound states as a function of $\lambda$ for $\mathrm{Li}+\mathrm{CaH}(J=0)$. The bottom panel shows an expanded view with a state approaching threshold, crossed by several steeper states. The dashed line has been added to follow this state through the avoided crossings and help guide the eye.

Next we consider higher values of $J$, which correspond to higher partial waves at the lowest rotational threshold. Figure 4 shows histograms of NNS for $J=1,3$ and 8. These notably do not show the linear level repulsion at small spacings expected for complete chaos. Instead there appears to be a finite probability of zero spacing. The corresponding Brody parameters are in the region of 0.4. Although at first sight this suggests a substantially reduced degree of chaos compared to $J=0$, such a distribution can also occur for two overlapping but noninteracting chaotic spectra [57,62]. This suggests that there is some form of symmetry or good quantum number present in the system. However, we have already taken account of all rigorous symmetries, so the quantity concerned must be only approximately conserved. On a finer scale, the NNS distribution does indeed show some limited level repulsion.

The nearly conserved quantum number can be understood in the body-fixed reference frame, rather than the space-fixed frame that we use in the coupled-channel calculations. It is the projection of the total angular momentum $J$ (or equivalently the diatom rotation $j$ ) onto the intermolecular axis, which is well known in studies of atom-diatom Van der Waals complexes [34] and is given the symbol $K$. It can take values from $-J$ to $+J$ in integer steps. Blocks of the Hamiltonian with different $K$ are 

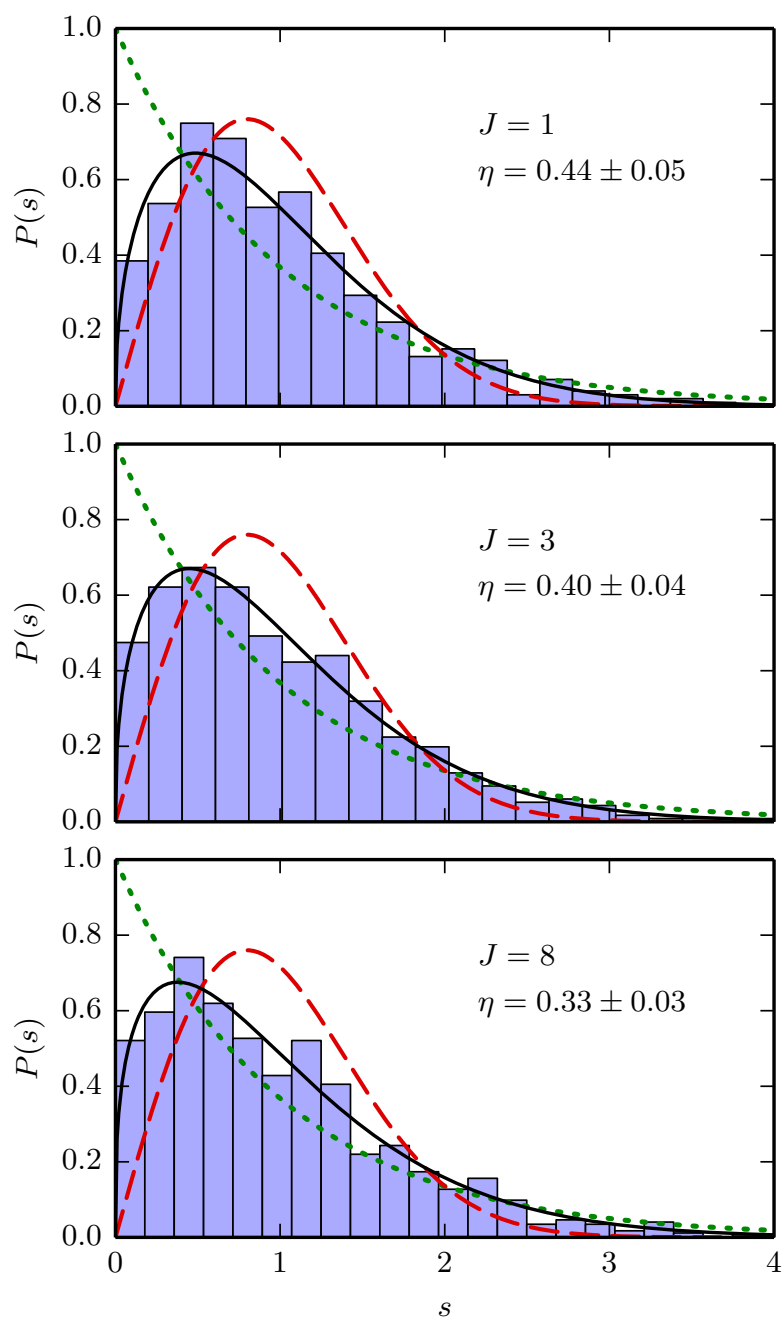

FIG. 4. (Color online) Nearest-neighbor spacing distributions for $\mathrm{Li}+\mathrm{CaH}: J=1(0.5 \leq \lambda \leq 1.5), J=3(0.8 \leq \lambda \leq 1.4)$, and $J=8(0.8 \leq \lambda \leq 1.3)$. Lines are as in Fig. 2 .

coupled only by Coriolis terms in the body-frame representation of the centrifugal motion; these Coriolis terms are very small compared to the potential anisotropy in the well region, so the Hamiltonian can be considered to be nearly block-diagonal with blocks labeled by $|K|$ and parity.

It is possible to carry out coupled-channel calculations in the body-fixed frame, neglecting the Coriolis terms offdiagonal in $K$. This makes the problem block-diagonal and is known as helicity decoupling; it is often an effective technique for calculations of atom-diatom bound states [34]. Figure 5(a) and (b) show NNS distributions for the separate $K=0$ and $|K|=1$ blocks. There is no further hidden symmetry and so the NNS distributions are once again close to the Wigner-Dyson limit. Figure 5(c) shows the statistics for the superposition of the two individual level sequences. This last case is close to that of two GOE level sequences which overlap but are not cou-
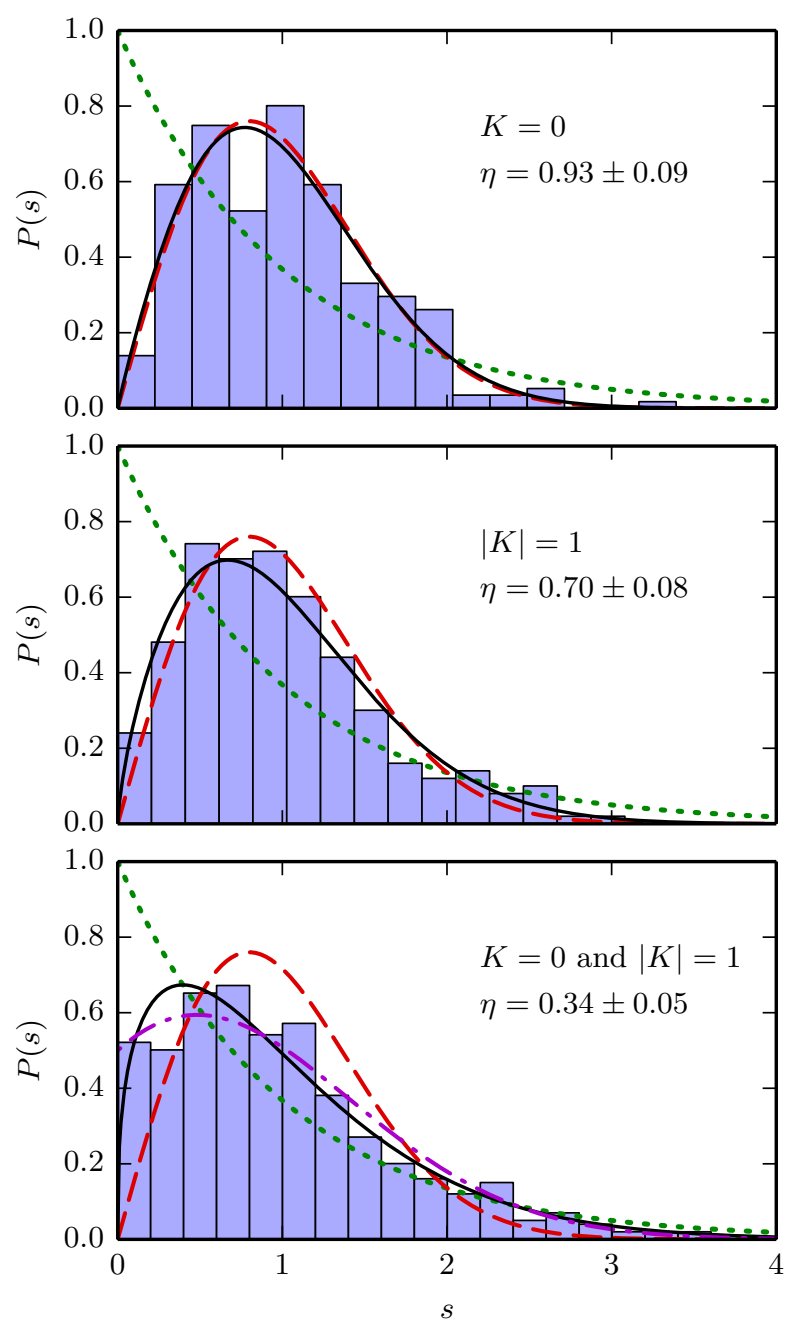

FIG. 5. (Color online) NNS distributions for $\mathrm{Li}+\mathrm{CaH}(J=1$, $0.5 \leq \lambda \leq 1.5)$ using the helicity-decoupling approximation. $K=0 ;|K|=1 ; K=0$ and $|K|=1$. Lines are as in Fig. 2, magenta dash-dot line in the bottom panel is the predicted NNS distribution for two overlapping GOEs.

pled. The resulting NNS distribution can be obtained from equation (3.69) of [22], and is also shown in Fig. $5(\mathrm{c})$. It differs from the Wigner-Dyson distribution most obviously in that it does not vanish at zero spacing. It is in good agreement with the results from helicity decoupling calculations and explains the qualitative behavior of the $J \neq 0$ distributions in Fig. 4.

The remaining differences between Fig. 4(a) and Fig. 5 (c) are due to the Coriolis terms. The quantitative effect of these terms on the statistics is beyond the scope of this paper, but it is nevertheless informative to look at the pattern of bound states near threshold. Figure 6 shows bound states for $J=1$ within $10 \mathrm{~cm}^{-1}$ of threshold for a small range of $\lambda$, both from a full calculation and within the helicity decoupling approximation. The $K=0$ levels for $J=1$ are only slightly shifted from 


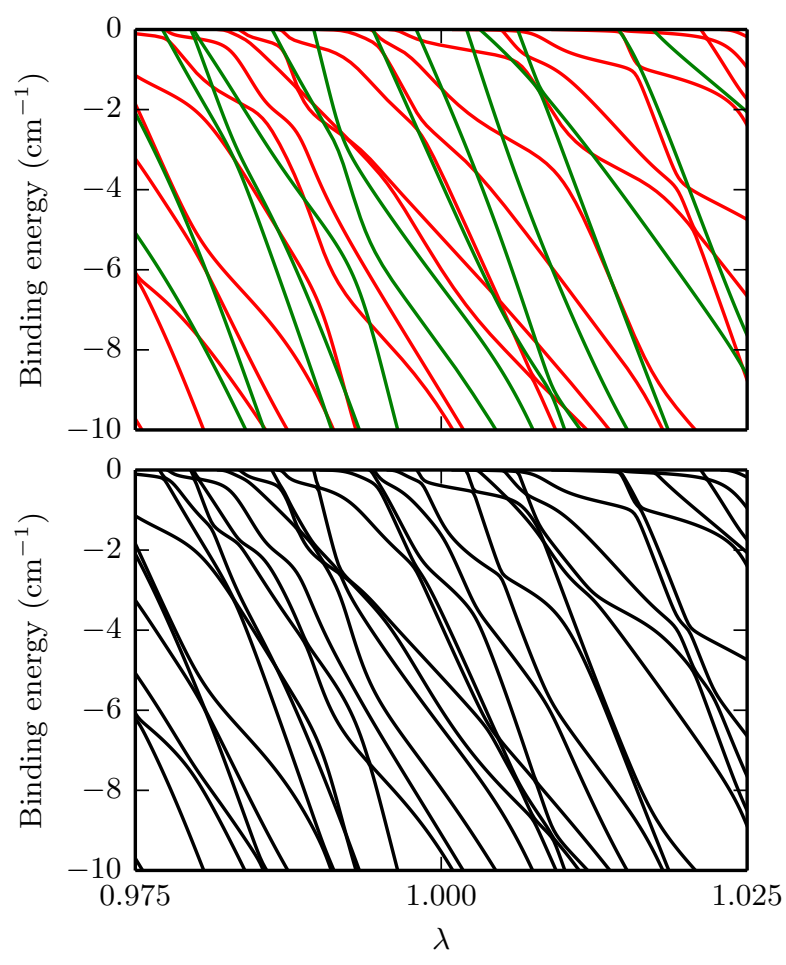

FIG. 6. (Color online) Near-threshold bound states as a function of $\lambda$ for $\mathrm{Li}+\mathrm{CaH}(J=1)$ : using the helicity-decoupling approximation (top; red: $K=0$, green: $|K|=1$ ); full calculation (bottom).

the levels for $J=0$ (top panel of Fig. 3). The $|K|=1$ levels are quite different but show the same qualitative features of many avoided crossings of a wide variety of strengths. However, in the helicity decoupling approximation, levels with one value of $|K|$ do not interact with those of different $|K|$; this gives rise to a large number of true crossings, producing an NNS distribution with finite probability at zero spacing. In the full calculation, which takes account of the Coriolis coupling between the blocks, the overall pattern of levels is similar but there are now narrow avoided crossings between levels of different $|K|$. These are typically much narrower than those between levels of the same $|K|$. This confirms our picture of a nearly conserved quantum number, with only weak coupling between states of different $|K|$.

\section{B. $\mathbf{L i}+\mathbf{C a F}$}

The second system we consider is $\mathrm{Li}+\mathrm{CaF}$. The rotational constant for $\mathrm{CaF}$ is about 13 times smaller than that for $\mathrm{CaH}$, while the potential surface is quite similar. The ratio of the anisotropy to $b$ is thus significantly greater for $\mathrm{CaF}$ than for $\mathrm{CaH}$. This stronger effective coupling might be expected to give equal or greater amounts of chaos for $\mathrm{Li}+\mathrm{CaF}$ compared to $\mathrm{Li}+\mathrm{CaH}$.
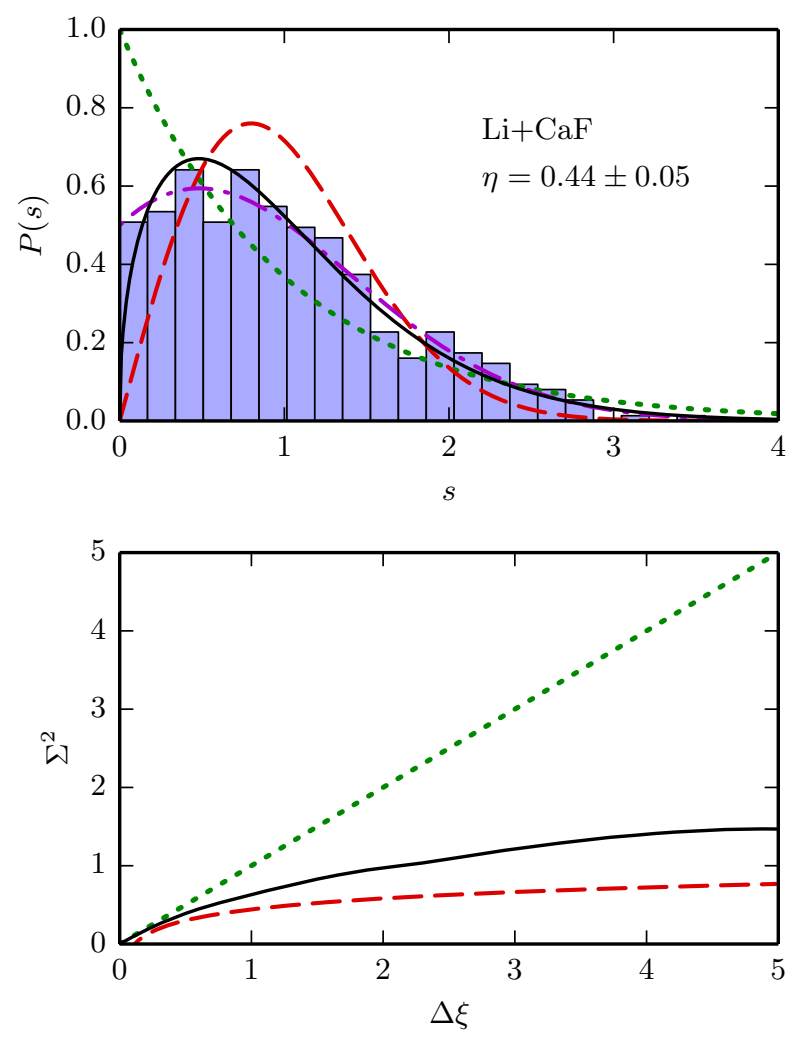

FIG. 7. (Color online) Calculated statistics for $\mathrm{Li}+\mathrm{CaF}$ $(J=0,0.8 \leq \lambda \leq 1.4)$ : NNS distribution, and level number variance. Lines are as in previous figures.

We have performed coupled-channel calculations for $\mathrm{Li}+\mathrm{CaF}(J=0)$ using the same methods as for $\mathrm{Li}+\mathrm{CaH}$, but with a larger basis set because of the smaller value of $b$. Figure 7 shows the resulting level statistics. Remarkably, the NNS distribution does not appear to show level repulsion, even for $J=0$, but neither does it resemble a Poisson distribution; the fitted Brody parameter is only 0.44. Once again the distribution bears a close resemblance to the case of two overlapping but noninteracting GOE spectra as discussed for the helicitydecoupled $J=1$ case for $\mathrm{Li}+\mathrm{CaH}$. This again hints at the possibility of some unexpected partially good quantum number, but in this $J=0$ case it cannot be the projection $|K|$. The level number variance for $\mathrm{Li}+\mathrm{CaF}$ $(J=0)$ is also some way from the GOE prediction, although it does level off at high spacings, in contrast to that in other near-chaotic examples [27, 28].

Figure 8 shows the binding energies of near-threshold levels for $\mathrm{Li}+\mathrm{CaF}(J=0)$ as a function of $\lambda$. It may be seen that some bound states have very steep energy gradients with respect to $\lambda$ and that these states interact weakly with those with shallower gradients [63]. In this respect the pattern shows a clear systematic difference from that observed for $\mathrm{Li}+\mathrm{CaH}$ (Fig. 3), where all $J=0$ states appeared significantly coupled and the levels could 


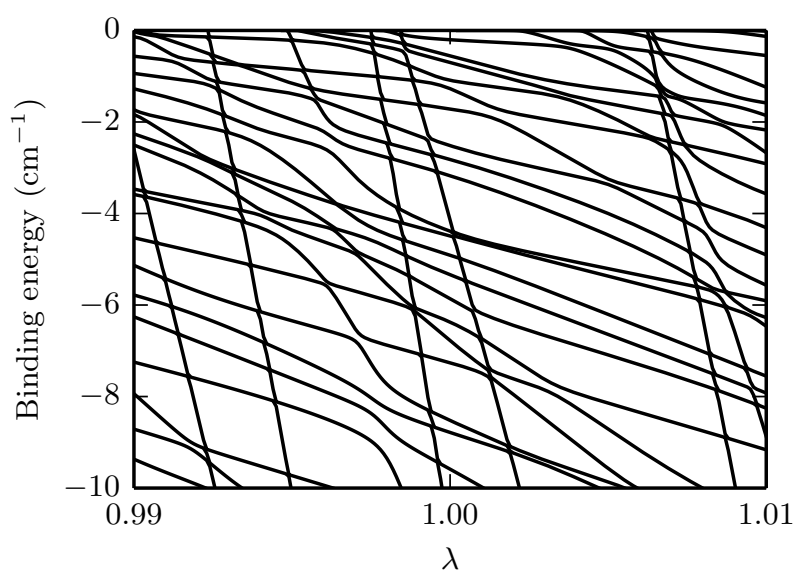

FIG. 8. Near-threshold bound states as a function of $\lambda$ for $\mathrm{Li}+\mathrm{CaF}(J=0)$.

not easily be separated into classes.

There has been a great deal of work on the energy levels of atom-diatom systems, largely aimed at understanding the dynamics of Van der Waals complexes [34]. For low anisotropies, the diatomic molecule executes hindered rotation in the complex, and the resulting internal rotation is only weakly coupled to the intermolecular stretching motion. However, when the effective anisotropy is comparable to or larger than the diatom rotational constant, there is significant mixing of diatom rotational states. As the anisotropy increases further, the internal rotation is transformed into a bending vibration of the triatomic molecule. Correlation diagrams showing how this transition occurs have been given in ref. 34 for complexes with both linear and non-linear equilibrium geometries. The low-lying levels of a non-linear species such as Li$\mathrm{CaH}$ or Li-CaF eventually execute low-amplitude bending vibrations about their non-linear equilibrium. The degree of mixing between bending and stretching degrees of freedom typically depends on their relative frequencies: if the bending is either much faster or much slower than the stretching then the modes can be separated in a Born-Oppenheimer sense [64-66], but if the frequencies are comparable then they are strongly mixed.

The situation is more complicated for highly excited states, such as the near-dissociation states that give rise to Feshbach resonances in the present work. Some highly excited states have unstructured nodal patterns that fill the energetically accessible space, but there are others with simple nodal patterns that sample restricted regions of space [67-70]. However, the paths along which such states are localized may be complicated ones that do not correspond to obvious quantum numbers. Because of this, it may be difficult to identify the specific nearly conserved quantity that divides the states in Fig. 8 into separate classes. Nevertheless, the level statistics appear to indicate that such a quantity exists.

\section{Variable rotational constant}

To understand better the puzzling difference between $\mathrm{Li}+\mathrm{CaH}$ and $\mathrm{Li}+\mathrm{CaF}$, we attempt to interpolate between our two systems and extrapolate beyond them. Since the two potential energy surfaces are so similar, we use the surface and reduced mass for $\mathrm{Li}+\mathrm{CaH}$ throughout this section, and vary the rotational constant. We increase the number of rotational basis functions from 55 to 350 as $b$ decreases from 100 to $0.01 \mathrm{~cm}^{-1}$ to obtain converged level positions.

Figure 9 shows the fitted Brody parameter as a function of rotational constant. This shows an astonishing structure. It can be seen that $b_{\mathrm{CaH}}$ lies in a relatively wide region from 0.7 to $7 \mathrm{~cm}^{-1}$ where $\eta$ is near unity, so that the systems can be said to be chaotic. Towards lower rotational constant the fitted Brody parameter falls sharply to about 0.4 in the region between 0.2 and 0.4 $\mathrm{cm}^{-1}$ - in which $b_{\mathrm{CaF}}$ lies - but it then rises rapidly back to values near unity for $0.05 \mathrm{~cm}^{-1}<b<0.08 \mathrm{~cm}^{-1}$ before beginning to fall slowly again. At higher values of $b$, there is another steep and narrow trough centered around $12 \mathrm{~cm}^{-1}$, followed by a steady decrease towards zero as the angular and radial motions become increasingly uncoupled.

Figure 10 shows statistics for sample values of the rotational constant $b$. Those for the lowest value, $b=0.05$ $\mathrm{cm}^{-1}$ [Fig. 10(a)] show almost perfect agreement with the GOE predictions for both NNS distribution and level number variance; the fitted Brody parameter is $\eta=0.97 \pm 0.06$. The second value, $b=0.25 \mathrm{~cm}^{-1}$, is close to $b_{\mathrm{CaF}}=0.339 \mathrm{~cm}^{-1}$, but the NNS distribution [Fig. 10(b)] differs from that seen for $\mathrm{Li}+\mathrm{CaF}$ in Fig. 7(a), with noticeably more level repulsion despite a lower $\eta . \quad b=1.0 \mathrm{~cm}^{-1}$ [Fig. 10(c)] is within the same region of high $\eta$ as $b_{\mathrm{CaH}}$ and also shows clear signs of chaos. $b=12.5 \mathrm{~cm}^{-1}$ [Fig. 10(d)] is located in a narrow trough of low $\eta$ and the statistics appear to be similar to case (b). $b=21 \mathrm{~cm}^{-1}$ [Fig. 10(e)] is located above the trough in $\eta$; the Brody parameter is only 0.8 but the statistics show all the qualitative features expected of a chaotic system. The statistics for $b=50 \mathrm{~cm}^{-1}$ [Fig. 10(f)] show an NNS distribution that is close to Poissonian $(\eta=0.16)$ because the rotational constant is large enough for the rotational and stretching motions to be significantly decoupled and the conditions for chaos no longer exist. However, the number variance does not rise linearly as in the Poisson case; instead it reaches a peak and turns downwards. The inset shows that, on a larger scale, this is the first in a complex series of oscillations, which we attribute to the onset of regularity and do not interpret further in this paper.

The presence of oscillations in the Brody parameter in Fig. 9 is puzzling. The argument given in section IV B above would predict a single maximum in the Brody parameter when the effective bending and stretching frequencies are comparable for near-threshold states, dropping off when the frequencies are very different. Fig. 9 


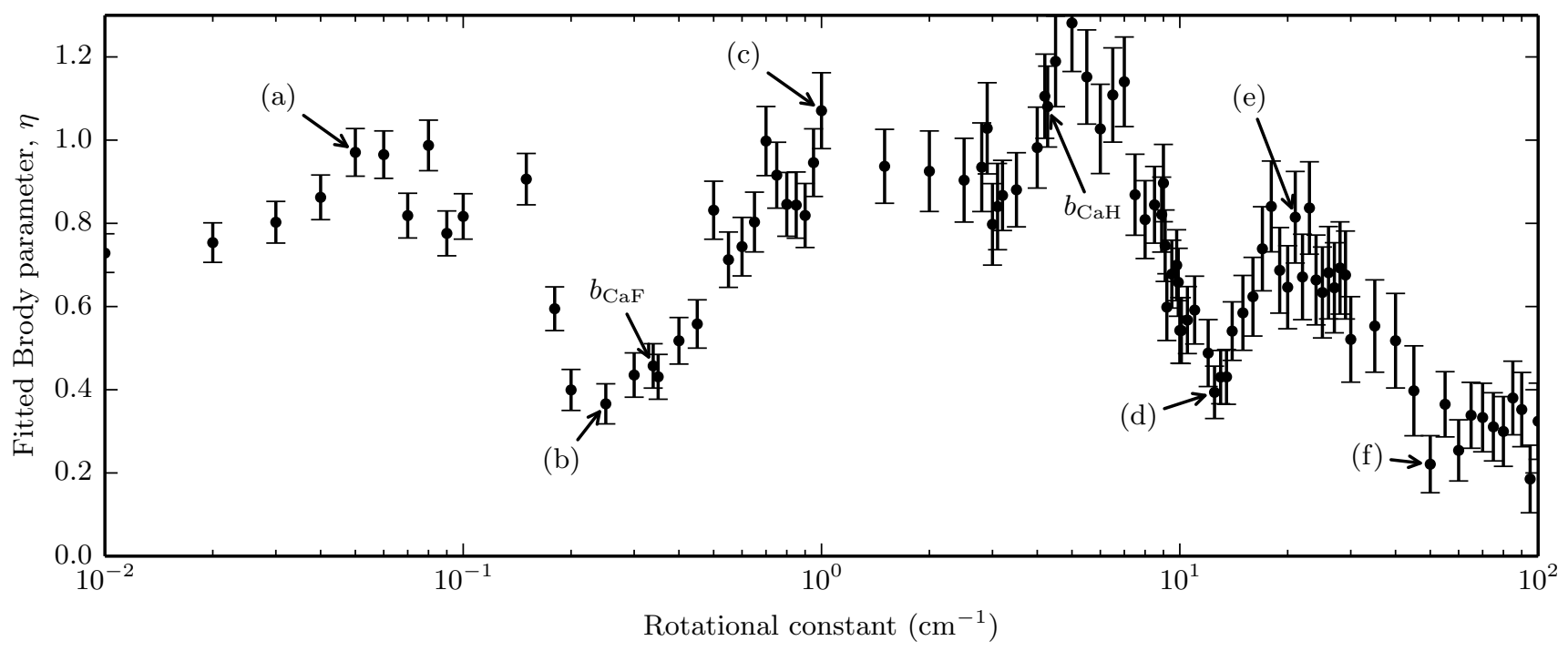

FIG. 9. Rotational constant dependence of fitted Brody parameter for $\mathrm{Li}+\mathrm{CaH}(J=0)$. Labeled points are: (a) 0.05, (b) 0.25, (c) 1.0, (d) 12.5, (e) 21, (f) $50 \mathrm{~cm}^{-1} \cdot b_{\mathrm{CaF}}$ and $b_{\mathrm{CaH}}$ are also indicated.

does appear to show such a maximum, but the argument does not explain the deep minima that seem to be present either side of it. It is possible that the flanking maxima correspond to effective bending frequencies that match the stretching frequencies for different stretching states, or that are rational multiples of effective stretching frequencies.

\section{CONCLUSIONS}

We have carried out calculations on threshold and near-threshold bound states of atom + rigid-rotor models of $\mathrm{Li}+\mathrm{CaH}$ and $\mathrm{Li}+\mathrm{CaF}$, and performed statistical analysis of the resulting level sequences. For $\mathrm{Li}+\mathrm{CaH}$ with zero total angular momentum we have found the strongest signs of chaos yet observed for a realistic ultracold collision system in either theory or experiment. However, for non-zero total angular momentum we found a nearly good quantum number which we identified as the body-fixed projection $K$ of the total angular momentum $J$. The presence of this nearly conserved quantity significantly alters the overall statistics, but the statistics for individual values of $K$ still show chaotic behavior. The superposition of two nearly independent level sequences in the $J=1$ case produces an NNS distribution that is distinct from the Poisson, Wigner-Dyson and Brody distributions.

The ratio of the anisotropy to the diatom rotational constant is significantly larger for $\mathrm{Li}+\mathrm{CaF}$ than for $\mathrm{Li}+\mathrm{CaH}$. Nevertheless, contrary to expectation, $\mathrm{Li}+\mathrm{CaF}$ shows less strongly chaotic behavior even for $J=0$. The similarity of the statistics with the case of $\mathrm{Li}+\mathrm{CaH}$ $(J=1)$ suggests the presence of another nearly good quantum number in $\mathrm{Li}+\mathrm{CaF}$. The emergence of this quantum number may be related to an adiabatic separation between a slow bending vibration and a faster intermolecular stretch.

Finally, we have investigated how the statistics change between and beyond our two systems by varying the rotational constant with a fixed potential. We observe astonishing fluctuations in the levels of chaos in the system. It thus cannot even be assumed that a system that is partway between two closely related chaotic systems will itself be chaotic. The origin of this surprising effect is unclear. One possibility is that stronger chaos emerges when the bending and stretching frequencies are close to rational multiples of one another.

This study has demonstrated that the relationship between coupling strength and chaos is complicated. Starting from a chaotic system and increasing the strength of a coupling does not necessarily lead to an increase in chaos. This should not be surprising in principle: if a single term in a hamiltonian becomes dominant, that term defines nearly good quantum numbers for the system. The superposition of nearly independent level sequences for different values of the nearly good quantum numbers then produces non-chaotic statistics.

It is clear that there is much to be learned from studying chaos in ultracold collisions and high-lying bound states of atoms and molecules. Statistical analysis can provide valuable insight when the spectra are too complex for direct analysis. However, this study has highlighted that deviations from chaotic behavior can be difficult to predict, even in apparently simple systems. Future work will focus on the origins of chaotic and nonchaotic statistics in increasingly complex systems. One question of particular importance is whether, in real sys- 

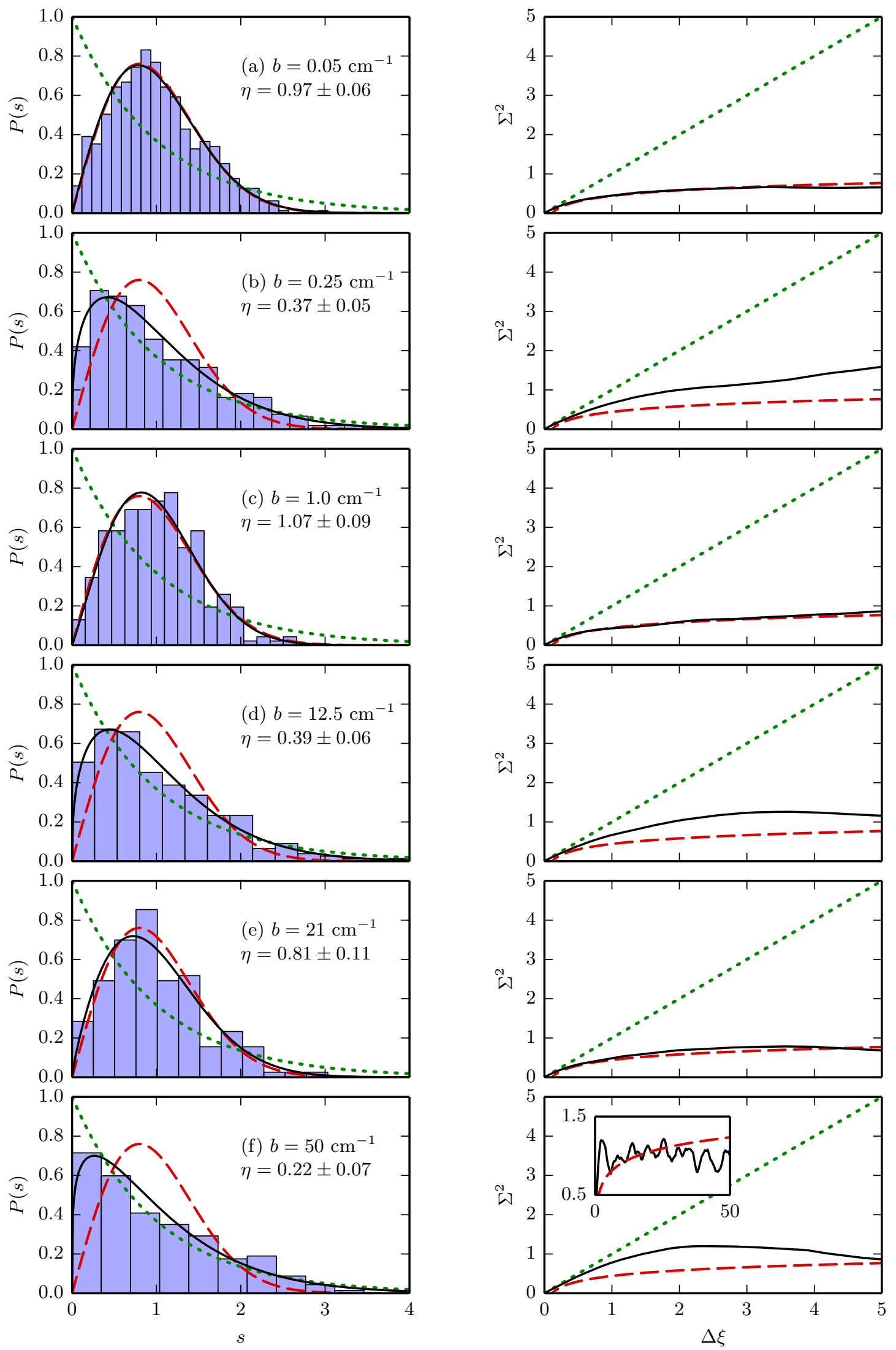

FIG. 10. (Color online) Examples of the NNS distribution (left) for values of rotational constant highlighted in Fig. 9, and the corresponding level number variance (right). Lines are as in previous figures. 
tems, all degrees of freedom are involved in the chaotic behavior, or whether there is a hierarchy of couplings that leaves some degrees of freedom uninvolved. Our results for $\mathrm{Li}+\mathrm{CaH}(J=1)$ represent a particularly simple example of a case with a clear hierarchy.

\section{ACKNOWLEDGMENTS}

This work has been supported by the UK Engineering and Physical Sciences Research Council (grant EP/I012044/1). The authors are grateful to Timur Tscherbul, Jacek Kłos and Alexei Buchachenko for supplying the potential energy surface used for $\mathrm{Li}+\mathrm{CaH}$.
[1] C. Chin, R. Grimm, E. Tiesinga, and P. S. Julienne, "Feshbach resonances in ultracold gases," Rev. Mod. Phys. 82, 1225 (2010).

[2] T. Takekoshi, M. Debatin, R. Rameshan, F. Ferlaino, R. Grimm, H.-C. Nägerl, C. R. Le Sueur, J. M. Hutson, P. S. Julienne, S. Kotochigova, and E. Tiemann, "Towards the production of ultracold ground-state RbCs molecules: Feshbach resonances, weakly bound states, and coupled-channel models," Phys. Rev. A 85, 032506 (2012).

[3] M. Berninger, A. Zenesini, B. Huang, W. Harm, H.-C. Nägerl, F. Ferlaino, R. Grimm, P. S. Julienne, and J. M. Hutson, "Feshbach resonances, weakly bound molecular states and coupled-channel potentials for cesium at high magnetic field," Phys. Rev. A 87, 032517 (2013).

[4] P. S. Julienne and J. M. Hutson, "Contrasting the wide Feshbach resonances in ${ }^{6} \mathrm{Li}$ and ${ }^{7} \mathrm{Li}$," Phys. Rev. A 89 , 052715 (2014).

[5] D. J. Frantzeskakis, "Dark solitons in atomic BoseEinstein condensates: from theory to experiments," J. Phys. A 43, 213001 (2010).

[6] T. Köhler, K. Góral, and P. S. Julienne, "Production of cold molecules via magnetically tunable Feshbach resonances," Rev. Mod. Phys. 78, 1311 (2006).

[7] T. Kraemer, M. Mark, P. Waldburger, J. G. Danzl, C. Chin, B. Engeser, A. D. Lange, K. Pilch, A. Jaakkola, H. C. Nägerl, and R. Grimm, "Evidence for Efimov quantum states in an ultracold gas of caesium atoms," Nature 440, 315 (2006).

[8] B. Huang, L. A. Sidorenkov, R. Grimm, and J. M. Hutson, "Observation of the second triatomic resonance in Efimov's scenario," Phys. Rev. Lett. 112, 190401 (2014).

[9] M. Lu, N. Q. Burdick, S. H. Youn, and B. L. Lev, "Strongly dipolar Bose-Einstein condensate of dysprosium," Phys. Rev. Lett. 107, 190401 (2011).

[10] B. Pasquiou, E. Maréchal, L. Vernac, O. Gorceix, and B. Laburthe-Tolra, "Thermodynamics of a Bose-Einstein condensate with free magnetization," Phys. Rev. Lett. 108, 045307 (2012).

[11] K. Aikawa, A. Frisch, M. Mark, S. Baier, A. Rietzler, R. Grimm, and F. Ferlaino, "Bose-Einstein condensation of erbium," Phys. Rev. Lett. 108, 210401 (2012).

[12] K. Baumann, N. Q. Burdick, M. Lu, and B. L. Lev, "Observation of low-field Fano-Feshbach resonances in ultracold gases of dysprosium," Phys. Rev. A 89, 020701 (2014).

[13] T. Maier, I. Ferrier-Barbut, H. Kadau, M. Schmitt, M. Wenzel, C. Wink, T. Pfau, K. Jachymski, and P. S. Julienne, "Broad universal Feshbach resonances in the chaotic spectrum of dysprosium atoms," Phys. Rev. A 92, 060702 (2015).
[14] A. Frisch, M. Mark, K. Aikawa, S. Baier, R. Grimm, A. Petrov, S. Kotochigova, G. Quéméner, M. Lepers, O. Dulieu, and F. Ferlaino, "Ultracold dipolar molecules composed of strongly magnetic atoms," Phys. Rev. Lett. 115, 203201 (2015).

[15] K.-K. Ni, S. Ospelkaus, M. H. G. de Miranda, A. Pe'er, B. Neyenhuis, J. J. Zirbel, S. Kotochigova, P. S. Julienne, D. S. Jin, and J. Ye, "A high phase-space-density gas of polar molecules in the rovibrational ground state," Science 322, 231 (2008).

[16] S. Ospelkaus, K.-K. Ni, D. Wang, M. H. G. de Miranda, B. Neyenhuis, G. Quéméner, P. S. Julienne, J. L. Bohn, D. S. Jin, and J. Ye, "Quantum-state controlled chemical reactions of ultracold KRb molecules," Science 327, 853 (2010).

[17] L. P. Parazzoli, N. J. Fitch, P. S. Żuchowski, J. M. Hutson, and H. J. Lewandowski, "Large effects of electric fields on atom-molecule collisions at millikelvin temperatures," Phys. Rev. Lett. 106, 193201 (2011).

[18] T. Takekoshi, L. Reichsöllner, A. Schindewolf, J. M. Hutson, C. R. Le Sueur, O. Dulieu, F. Ferlaino, R. Grimm, and H.-C. Nägerl, "Ultracold dense samples of dipolar RbCs molecules in the rovibrational and hyperfine ground state," Phys. Rev. Lett. 113, 205301 (2014).

[19] P. K. Molony, P. D. Gregory, Z. Ji, B. Lu, M. P. Köppinger, C. R. Le Sueur, C. L. Blackley, J. M. Hutson, and S. L. Cornish, "Creation of ultracold ${ }^{87} \mathrm{Rb}^{133} \mathrm{Cs}$ molecules in the rovibrational ground state," Phys. Rev. Lett. 113, 255301 (2014).

[20] A. Frisch, M. Mark, K. Aikawa, F. Ferlaino, J. L. Bohn, C. Makrides, A. Petrov, and S. Kotochigova, "Quantum chaos in ultracold collisions of gas-phase erbium atoms," Nature 507, 475 (2014).

[21] M. L. Mehta, Random Matrices, 2nd ed. (Academic Press, 1991).

[22] T. Guhr, A. Müller-Groeling, and H. A. Weidenmüller, "Random matrix theories in quantum physics: common concepts," Phys. Rep. 299, 189 (1998).

[23] E. P. Wigner, "Characteristic vectors of bordered matrices with infinite dimensions," Ann. Math. Second Series, 62, pp. 548 (1955).

[24] F. J. Dyson, "Statistical theory of the energy levels of complex systems. I," J. Math. Phys. 3, 140 (1962).

[25] M. Mayle, B. P. Ruzic, and J. L. Bohn, "Statistical aspects of ultracold resonant scattering," Phys. Rev. A 85, 062712 (2012).

[26] M. Mayle, G. Quéméner, B. P. Ruzic, and J. L. Bohn, "Scattering of ultracold molecules in the highly resonant regime," Phys. Rev. A 87, 012709 (2013).

[27] T. Maier, H. Kadau, M. Schmitt, M. Wenzel, I. FerrierBarbut, T. Pfau, A. Frisch, S. Baier, K. Aikawa, L. Chomaz, M. J. Mark, F. Ferlaino, C. Makrides, 
E. Tiesinga, A. Petrov, and S. Kotochigova, "Emergence of chaotic scattering in ultracold Er and Dy," Phys. Rev. X 5, 041029 (2015).

[28] K. Jachymski and P. S. Julienne, "Chaotic scattering in the presence of a dense set of overlapping Feshbach resonances," Phys. Rev. A 92, 020702 (2015).

[29] O. Bohigas, M. J. Giannoni, and C. Schmit, "Characterization of chaotic quantum spectra and universality of level fluctuation laws," Phys. Rev. Lett. 52, 1 (1984).

[30] J. Mur-Petit and R. A. Molina, "Spectral statistics of molecular resonances in erbium isotopes: How chaotic are they?" Phys. Rev. E 92, 042906 (2015).

[31] D. G. Green, C. L. Vaillant, M. D. Frye, M. Morita, and J. M. Hutson, "Quantum chaos in ultracold collisions between $\mathrm{Yb}\left({ }^{1} \mathrm{~S}_{0}\right)$ and $\mathrm{Yb}\left({ }^{3} \mathrm{P}_{2}\right)$," Phys. Rev. A 93, 022703 (2016).

[32] M. L. González-Martínez and P. S. Żuchowski, "Magnetically tunable Feshbach resonances in Li+Er," Phys. Rev. A 92, 022708 (2015).

[33] R. B. Bernstein, ed., Atom-Molecule Collision Theory: a Guide for the Experimentalist (Plenum Press, New York, 1979).

[34] J. M. Hutson, "An introduction to the dynamics of Van der Waals molecules," in Advances in Molecular Vibrations and Collision Dynamics, Vol. 1A (JAI Press, Greenwich, Connecticut, 1991) pp. 1-45.

[35] J. M. Hutson, "Coupled-channel methods for solving the bound-state Schrödinger equation," Comput. Phys. Commun. 84, 1 (1994).

[36] J. M. Hutson and S. Green, "MOLSCAT computer program, version 14," distributed by Collaborative Computational Project No. 6 of the UK Engineering and Physical Sciences Research Council (1994).

[37] J. M. Hutson, "BOUND computer program, version 5," distributed by Collaborative Computational Project No. 6 of the UK Engineering and Physical Sciences Research Council (1993).

[38] M. T. Hummon, T. V. Tscherbul, J. Kłos, H.-I. Lu, E. Tsikata, W. C. Campbell, A. Dalgarno, and J. M. Doyle, "Cold $\mathrm{N}+\mathrm{NH}$ collisions in a magnetic trap," Phys. Rev. Lett. 106, 053201 (2011).

[39] H.-I. Lu, I. Kozyryev, B. Hemmerling, J. Piskorski, and J. M. Doyle, "Magnetic trapping of molecules via optical loading and magnetic slowing," Phys. Rev. Lett. 112, 113006 (2014).

[40] T. V. Tscherbul, J. Kłos, and A. A. Buchachenko, "Ultracold spin-polarized mixtures of ${ }^{2} \Sigma$ molecules with $S$ state atoms: Collisional stability and implications for sympathetic cooling," Phys. Rev. A 84, 040701 (2011).

[41] H.-J. Werner, P. J. Knowles, G. Knizia, F. R. Manby, M. Schütz, P. Celani, T. Korona, R. Lindh, A. Mitrushenkov, G. Rauhut, K. R. Shamasundar, T. B. Adler, R. D. Amos, A. Bernhardsson, A. Berning, D. L. Cooper, M. J. O. Deegan, A. J. Dobbyn, F. Eckert, E. Goll, C. Hampel, A. Hesselmann, G. Hetzer, T. Hrenar, G. Jansen, C. Köppl, Y. Liu, A. W. Lloyd, R. A. Mata, A. J. May, S. J. McNicholas, W. Meyer, M. E. Mura, A. Nicklass, D. P. O’Neill, P. Palmieri, K. Pflüger, R. Pitzer, M. Reiher, T. Shiozaki, H. Stoll, A. J. Stone, R. Tarroni, T. Thorsteinsson, M. Wang, and A. Wolf, "MOLPRO, version 2010.1, a package of ab initio programs," (2015), see http://www.molpro.net.
[42] T. H. Dunning, Jr., "Gaussian basis sets for use in correlated molecular calculations. I. The atoms boron through neon and hydrogen," J. Chem. Phys. 90, 1007 (1989).

[43] R. A. Kendall, T. H. Dunning, and R. J. Harrison, "Electron affinities of the first-row atoms revisited. Systematic basis sets and wave functions," J. Chem. Phys. 96, 6796 (1992).

[44] J. Koput and K. A. Peterson, "Ab initio potential energy surface and vibrational-rotational energy levels of $\mathrm{X}^{2} \Sigma^{+}$ CaOH," J. Phys. Chem. A 106, 9595 (2002).

[45] S. F. Boys and F. Bernardi, "The calculation of small molecular interactions by the differences of separate total energies. Some procedures with reduced errors," Mol. Phys. 19, 553 (1970).

[46] L. A. Kaledin, J. C. Bloch, M. C. McCarthy, and R. W. Field, "Analysis and deperturbation of the $A^{2} \Pi$ and $B^{2} \Sigma^{+}$states of CaF," J. Mol. Spectrosc. 197, 289 (1999).

[47] T. A. Brody, J. Flores, J. B. French, P. A. Mellow, A. Pandey, and S. S. M. Wong, "Random-matrix physics: spectrum and strength fluctuations," Rev. Mod. Phys. 53, 385 (1981).

[48] M. T. Cvitaš, P. Soldán, J. M. Hutson, P. Honvault, and J. M. Launay, "Interactions and dynamics in $\mathrm{Li}+\mathrm{Li}_{2}$ ultracold collisions," J. Chem. Phys. 127, 074302 (2007).

[49] A. O. G. Wallis and J. M. Hutson, "Production of ultracold NH molecules by sympathetic cooling with Mg," Phys. Rev. Lett. 103, 183201 (2009).

[50] A. O. G. Wallis, E. J. J. Longdon, P. S. Żuchowski, and J. M. Hutson, "The prospects of sympathetic cooling of NH molecules with Li atoms," Eur. Phys. J. D 65, 151 (2011).

[51] Note that even $\lambda=1$ should not be interpreted as the true potential because there are significant uncertainties in the calculated potentials.

[52] J. M. Hutson, "FIELD computer program, version 1," (2011).

[53] M. H. Alexander and D. E. Manolopoulos, "A stable linear reference potential algorithm for solution of the quantum close-coupled equations in molecular scattering theory," J. Chem. Phys. 86, 2044 (1987).

[54] M. Mizushima, Theory of Rotating Diatomic Molecules (Wiley, New York, 1975).

[55] The anisotropy considered in Ref. [27] is based entirely on dispersion effects [71]. The spread of dispersion coefficients for different potential curves of Er-Er is $10 \%$ of their mean value, and that for Dy-Dy is $9 \%$ of their mean value.

[56] We observe only a fraction of the total number of levels in the system, so $n \ll N$.

[57] M. V. Berry and M. Robnik, "Semiclassical level spacings when regular and chaotic orbits coexist," J. Phys. A 17, 2413 (1984).

[58] F. Izrailev, "Quantum localization and statistics of quasienergy spectrum in a classically chaotic system," Phys. Lett. A 134, 13 (1988).

[59] T. Brody, "A statistical measure for the repulsion of energy levels," Lett. Nuovo Cimento 7, 482 (1973).

[60] R. J. Barlow, Statistics: A Guide to the Use of Statistical Methods in the Physical Sciences (Wiley, 1989).

[61] M. V. Berry and M. Tabor, "Level clustering in the regular spectrum," Proc. R. Soc. A 356, 375 (1977).

[62] N. Rosenzweig and C. E. Porter, "Repulsion of energy levels' in complex atomic spectra," Phys. Rev. 120, 1698 
(1960).

[63] Although visually there may seem to be relatively few steep states for $\mathrm{Li}+\mathrm{CaF}$, counting them with respect to $\lambda$ at fixed energy reveals that approximately $1 / 3$ of the levels are of this type.

[64] S. L. Holmgren, M. Waldman, and W. Klemperer, "Internal dynamics of van der Waals complexes. I. BornOppenheimer separation of radial and angular motion," J. Chem. Phys. 67, 4414 (1977).

[65] J. M. Hutson and B. J. Howard, "Spectroscopic properties and potential surfaces for atom-diatom van der Waals molecules," Mol. Phys. 41, 1123 (1980).

[66] J. M. Hutson and B. J. Howard, "Anisotropic intermolecular forces. I. Rare gas-hydrogen chloride systems," Mol. Phys. 45, 769 (1982).
[67] C. R. Le Sueur, J. R. Henderson, and J. Tennyson, "Gateway states and bath states in the vibrational spectrum of $\mathrm{H}_{3}^{+}$," Chem. Phys. Lett. 206, 429 (1993).

[68] G. G. de Polavieja, F. Borondo, and R. M. Benito, "Scars in groups of eigenstates in a classically chaotic system," Phys. Rev. Lett. 73, 1613 (1994).

[69] N. J. Wright and J. M. Hutson, "Regular and irregular vibrational states: Localized anharmonic modes in $\mathrm{Ar}_{3}$," J. Chem. Phys. 110, 902 (1999).

[70] N. J. Wright and J. M. Hutson, "Regular and irregular vibrational states: Localized anharmonic modes and transition-state spectroscopy of $\mathrm{Na}_{3}, "$ J. Chem. Phys. 112, 3214 (2000).

[71] A. Petrov, E. Tiesinga, and S. Kotochigova, "Anisotropy-induced Feshbach resonances in a quantum dipolar gas of highly magnetic atoms," Phys. Rev. Lett. 109, 103002 (2012). 